\title{
HIV-I Infections During Vaccine Trials: Identifying New Epitopes For Differential Diagnosis Of HIV-I Infections In The Face Of Vaccine -Induced Antibodies
}

\author{
Hana Golding*‡, James Needham and Surender Khurana
}

Address: Div. of Viral Products, Center for Biologics Evaluation and Research (CBER), FDA, Bethesda, MD, USA 20892

Email: Hana Golding* - goldingh@cber.FDA.gov

* Corresponding author ‡Presenting author

from 2005 International Meeting of The Institute of Human Virology Baltimore, USA, 29 August - 2 September 2005

Published: 8 December 2005

Retrovirology 2005, 2(Suppl I):S68 doi:I0.1 I86/I742-4690-2-SI-S68

Most of the HIV-1 vaccines under development contain multiple viral genes or proteins. As a result, many vaccinerecipients react positive in licensed HIV-1 detection assays. This will have negative impact on future efficacy trials of prophylactic HIV vaccines that require early detection of intercurrent HIV infections. It will also exclude all vaccinees from blood/plasma donations, and may contribute to other social harms. Therefore, it is important to design new strategies for vaccine trial participants that will clearly discriminate between vaccine-induced antibodies and true HIV-1 infection. We identified new HIV-1 epitopes that: 1) Do not contain important neutralizing or CTL epitopes, and can be omitted from future HIV vaccine candidates, 2) Recognized by antibodies from early HIV infected individuals, 3) Highly conserved among HIV-1 clades and subtypes. Using Phage Display libraries constructed from whole HIV-1 genome, combined with panning over antibodies from early seroconvertors, we identified new immunodominant epitopes, in the gp41 intracytoplasmic tail and in p6, which conform to the above criteria.

These peptides were used for the development of new HIV-1 EIA. To date the assay specificity and sensitivity are at $>99 \%$. Based on reactivity of several well-characterized panels of seroconvertors it was demonstrated that these peptides could detect antibodies within 4 weeks of HIV-1 infection. Testing of diverse serum samples ( $>2100)$ from around the world supports the utilization of our assay in detecting antibodies from infected individuals with clades A, B, C, D, E, F, and multiple recombinants. Importantly, testing of sera from HIV-1 vaccine trials gave mostly negative reactivity in our assay while scoring positive in one or more of the currently FDA-licensed HIV-1/2 EIA kits. Furthermore, our assay detected intercurrent HIV infections among vaccine recipients in 4 different vaccine trials. This assay could be added to the HIV detection kits used in prophylactic vaccine trials and blood/plasma collection centers. 